\title{
Interference Enhancement of the Internal Fields at Structural Scattering Resonances of a Coated Sphere
}

James A. Lock

Cleveland State University, j.lock@csuohio.edu

Follow this and additional works at: https://engagedscholarship.csuohio.edu/sciphysics_facpub

Part of the Physics Commons

How does access to this work benefit you? Let us know!

\section{Publisher's Statement}

This paper was published in Applied Optics and is made available as an electronic reprint with the permission of OSA. The paper can be found at the following URL on the OSA website: http://www.opticsinfobase.org/ao/abstract.cfm?URI=ao-29-21-3180. Systematic or multiple reproduction or distribution to multiple locations via electronic or other means is prohibited and is subject to penalties under law.

\section{Original Citation}

Lock, James A. "Interference Enhancement of the Internal Fields at Structural Scattering Resonances of a Coated Sphere." Applied Optics 29 (1990): 3180-3187.

\section{Repository Citation}

Lock, James A., "Interference Enhancement of the Internal Fields at Structural Scattering Resonances of a Coated Sphere" (1990). Physics Faculty Publications. 53.

https://engagedscholarship.csuohio.edu/sciphysics_facpub/53

This Article is brought to you for free and open access by the Physics Department at EngagedScholarship@CSU. It has been accepted for inclusion in Physics Faculty Publications by an authorized administrator of EngagedScholarship@CSU. For more information, please contact library.es@csuohio.edu. 


\title{
Interference enhancement of the internal fields at structural scattering resonances of a coated sphere
}

\author{
James A. Lock
}

\begin{abstract}
We examine the interior electromagnetic energy density of a coated nonabsorbing sphere at a number of scattering resonances. As is the case for an uncoated sphere, the interior energy density becomes large at the $a_{\ell}^{p}$ and $b_{\ell}^{p}$ resonances. It becomes especially large for $p-1$ values of the coating thickness at resonance. These $p-1$ enhancements are analogous to the interference maxima that occur in the intensity reflectance of two thin films in contact.
\end{abstract}

\section{Introduction}

In recent years, there have been many theoretical and experimental studies of the properties of the structural resonances in electromagnetic scattering by spherical and cylindrical particles. This type of resonance is called structural since it arises from a matching of the geometry of a single transverse electric or transverse magnetic multipole wave to the size and geometry of the particle, rather than resulting from atomic or molecular excitation of the particle material. Structural scattering resonances appear as strong narrow enhancements in the scattering cross section of the particle ${ }^{1-5}$ and as large interior field strengths near the particle surface ${ }^{6,7}$ when the scattering cross section and interior field strengths are considered as functions of the particle radius. The large interior energy density present at a structural scattering resonance has been experimentally detected by enhanced fluorescence excitation, ${ }^{8}$ Raman scattering, ${ }^{9}$ and enhanced rates of certain chemical reactions, ${ }^{10}$ and by various nonlinear processes such as laser emission ${ }^{11}$ and stimulated $\mathrm{Ra}$ man scattering. ${ }^{12}$ An increased heating rate at resonance has also been predicted. 6,13

Recently, the properties of structural scattering resonances of a coated sphere have been theoretically studied.14-18 The resonances of coated particles have a number of potential applications. For example, the resonance spectra of homogeneous spheres and cylinders are employed in diameter and refractive index characterizations of these particles. It might also be feasible to employ the resonance spectrum of a coated

The author is with Cleveland State University, Physics Department, Cleveland, Ohio 44115.

Received 7 March 1989.

0003-6935/90/213180-08\$02.00/0.

(C) 1990 Optical Society of America. cylinder for diameter and refractive index characterization of two-layer optical communication fibers. Similarly, the properties of a thick contamination layer on the surface of a homogeneous particle may be studied by the resonance spectroscopy of a coated particle. Also, coated sphere resonances have important implications for climatology research, since cloud and fog droplets encapsulating soot particles absorb more visible light than do homogeneous water droplets.

In this paper, we study the behavior of the interior electromagnetic energy density as a function of coating thickness at the structural scattering resonances of a coated sphere. Since many properties of the resonances of a coated particle are analogous to those of an uncoated particle, we recount a number of the properties of uncoated particle resonances in Sec. II. We examine the coated sphere resonances in Sec. III. We find an interesting property of coated sphere resonances that has no analog for an uncoated particle. It does, however, have an analog in the reflection of normally incident plane waves from two nonabsorbing thin films in contact. We examine this analogy in Sec. IV.

\section{Structural Scattering Resonances of an Uncoated Particle}

The scattered and interior partial wave amplitudes for a homogeneous sphere or cylinder may be written in the form (see, e.g., Ref. 19):

$$
\begin{aligned}
& a_{\ell}(x)=\frac{\alpha_{\ell}(x)}{\alpha_{\ell}(x)+i \gamma_{\ell}(x)} \\
& b_{\ell}(x)=\frac{\beta_{\ell}(x)}{\beta_{\ell}(x)+i \delta_{\ell}(x)} \\
& c_{\ell}(x)=\frac{i}{\alpha_{\ell}(x)+i \gamma_{\ell}(x)}
\end{aligned}
$$




$$
d_{\ell}(x)=\frac{i}{\beta_{\ell}(x)+i \delta_{\ell}(x)},
$$

respectively where $\alpha_{\ell}(x), \beta_{\ell}(x), \gamma_{\ell}(x)$, and $\delta_{\ell}(x)$ are sums of products of Riccati-Bessel functions, $A$ is the radius of the sphere or cylinder, $\lambda$ is the wavelength of the incident plane wave,

$$
k=\frac{2 \pi}{\lambda}
$$

is the incident wavevector, and

$$
x=k A
$$

is the size parameter of the particle. For a given partial wave $l$, the denominators of Eqs. (1) and (3) or of Eqs. (2) and (4) vanish at an infinite sequence of complex values of the size parameter. These poles of the partial wave scattering amplitudes in the complex $x$-plane are the source of scattering resonances. We denote the $p$-order of the $\ell$-th partial wave transverse magnetic and transverse electric scattering resonances by $a_{\ell}^{p}$ and $b_{\ell}^{p}$ respectively for $1 \leq p<\infty$. For a given partial wave, the scattering amplitude pole occurs at a larger distance from the real axis of the complex- $x$ plane as the resonance order $p$ increases and as the index of refraction of the particle decreases. ${ }^{20}$ As a result, the observed $a_{\ell}^{p}$ and $b_{\ell}^{p}$ resonances become weaker and broader for increasing $p$ and decreasing refractive index. For real $x$, the $a_{\ell}^{p}$ and $b_{\ell}^{p}$ resonances are centered at the size parameters $x_{p}$ for which

$$
\gamma_{\ell}\left(x_{p}\right)=0
$$

or

$$
\delta_{\ell}\left(x_{p}\right)=0,
$$

respectively.

Only the resonances for intermediate values of $p$ have been experimentally observed, and for a given size particle with a given refractive index, only one or two values of $p$ are accessible in any given experiment. The lowest $p$ resonances have not been observed since their extreme narrowness is beyond present spectrometer resolution and because they are strongly damped by small imperfections in the particle shape and by a small absorptive component of the index of refraction. ${ }^{2,9,21}$ Higher $p$ resonances are more stable against shape imperfections and absorption. However, when $p$ becomes sufficiently large, the resonances become too broad and weak to appear above the background provided by the nonresonant partial waves.

The physical mechanism responsible for the production of scattering resonances is apparent in the Debye expansion of scattering amplitudes $a_{\ell}(x)$ and $b_{\ell}(x)$, see, e.g., Refs. 22 and 23. The scattering amplitude for each radially propagating partial wave may be written in the Debye expansion as a diffractive component, plus a component representing reflection of the partial wave from the surface of the sphere, plus an infinite series of components representing transmission of the partial wave through the sphere following differing numbers of internal reflections from the surface. The interior amplitudes $c_{\ell}(x)$ and $d_{\ell}(x)$ may be expanded similarly. A scattering resonance occurs when each of the transmitted components for a single partial wave has the same phase and they all constructively interfere. ${ }^{23}$

It has often been stated that scattering resonances are a manifestation of tangentially propagating surface waves. ${ }^{1,2,4,5}$ Much care must be taken in making this statement since various authors have identified two different types of surface waves in the scattering by a transparent sphere. ${ }^{24,25}$ The first type of surface wave has been described in detail by Nussenzveig. ${ }^{22,26}$ It has a simple physical interpretation and it provides a large contribution to the van de Hulst term in the analysis of the glory. ${ }^{27} \mathrm{It}$, however, is not related to scattering resonances. ${ }^{28,29}$ The second type of surface wave has also been described in detail. $24,25,30,31 \mathrm{Al}$ though this second type of surface wave is associated with scattering resonances, it appears not to have a simple physical interpretation.

The angle-averaged electromagnetic energy density inside a nonconducting spherical particle is given by

$$
\epsilon(r)=\frac{1}{4 \pi} \int_{0}^{\pi} \sin \theta d \theta \int_{0}^{2 \pi} d \phi\left(\frac{n^{2} \epsilon_{0}}{2} \mathbf{E}^{*}(\mathbf{r}) \cdot \mathbf{E}(\mathbf{r})+\frac{1}{2 \mu_{0}} \mathbf{B}^{*}(\mathbf{r}) \cdot \mathbf{B}(\mathbf{r})\right)
$$

where $\mathbf{E}$ and $\mathbf{B}$ are the interior electric and magnetic fields, $\epsilon_{0}$ and $\mu_{0}$ are the permittivity and permeability of free space, and $n$ is the index of refraction of the particle. At the $a_{\ell}^{p}$ and $b_{\ell}^{p}$ resonances of a homogeneous sphere, the interior fields are dominated by the contribution of the $\ell$-th partial wave which is approximately proportional to the spherical Bessel function $j_{\ell}(n k r)$. The interior fields thus have $(2 p-1) / 4$ radial oscillations at the $a_{\ell}^{p}$ and $b_{\ell}^{p}$ resonances. ${ }^{9}$ Mathematically, the reason $\epsilon(r)$ can have such an enormous strength at resonance is that although the scattered partial wave amplitudes $a_{\ell}(x)$ and $b_{\ell}(x)$ of Eqs. ( 1 and 2 ) are constrained to magnitudes no greater than unity by conservation of energy (otherwose known as the unitarity of the scattering amplitude, see, e.g., Refs. 22, $32)$ the internal partial wave amplitudes $c_{\ell}(x)$ and $d_{\ell}(x)$ of Eqs. (3 and 4) are not subject to a unitarity constraint. Physically, since the interior of the particle is bounded, large amplitude standing waves which produce large energy densities can be set up inside the particle. Large energy densities cannot occur for the unbounded exterior region unless the scattered amplitude is superposed with the incident amplitude, as for example in the production of the spherical aberration caustic.

When the interior energy density in the $\theta=0^{\circ}, \theta=$ $180^{\circ}$ directions was calculated for an uncoated sphere at various scattering resonances in Ref. 33 , it was found that the energy density had $(2 p-1) / 4$ radial oscillations for the $b_{\ell}^{p}$ resonance and $(2 p+1) / 4$ oscillations for the $a_{\ell}^{p}$ resonance. This difference is not in conflict with behavior of the angle-averaged interior energy density. In the $\theta=0^{\circ}$ and $\theta=180^{\circ}$ directions, the Mie scattering angular functions $\pi_{\ell}(\theta)$ and $\tau_{\ell}(\theta)$ take on simple forms, and as a result, the $\ell$ partial wave contribution to the transverse electric field at these angles is 
proportional to $n k r j_{\ell}(n k r)$. There are $(2 p-1) / 4$ oscillations of this spherical Bessel function inside the sphere. The $\ell$-th partial wave contribution to the transverse magnetic field at $\theta=0^{\circ}, 180^{\circ}$ is proportional to $\left(n k r j_{\ell}(n k r)\right)^{\prime}$ which contains nearly equal and opposite amounts of $j_{\ell}(n k r)$ and $j_{\ell-1}(n k r)$. The interference of these two spherical Bessel functions creates the extra half oscillation of the field within the sphere in the $\theta=0^{\circ}, 180^{\circ}$ directions.

\section{Structural Scattering Resonances of a Coated Sphere}

As was the case for the uncoated sphere, the scattered partial wave amplitudes for the coated sphere may be written in the form given by Eqs. ( 1 and 2 ) and the interior partial wave amplitudes in both the core and coating may be written in forms similar to Eqs. (3 and 4). Conservation of energy again places an upper limit of unity on the exterior scattering amplitudes but the interior amplitudes are not constrained. For a coated nonabsorbing sphere, the $a_{\ell}^{p}$ and $b_{\ell}^{p}$ resonances again correspond to strong enhancements in both the scattering cross section and in the strength of the electromagnetic energy density in the coating and core. In the balance of this paper, we consider a plane wave of light incident on a coated sphere whose core is a nonabsorbing material of radius $A$ with real refractive index $n_{1}$ and whose coating is another nonabsorbing material of thickness $\Delta$ with real refractive index $n_{2}$. The overall radius of the composite particle is

$$
B=A+\Delta \text {. }
$$

The expressions for the interior and scattered partial wave amplitudes and for the angle-averaged electromagnetic energy density in the coating and core have been given in Ref. 18.

The core radii which produce the $a_{46}^{3}$ and $b_{46}^{3}$ scattering resonances were computed as a function of $\Delta$ for two different sets of refractive indices, $n_{1}=1.47$ and $n_{2}$ $=1.65$ (case I), and $n_{1}=1.47$ and $n_{2}=1.33$ (case II). The computer program employed in these computations was virtually identical to the program given in Ref. 34. In the limit $\Delta \rightarrow 0$, the core radii for the composite sphere resonances were found to approach the radii for the $n=1.47$ uncoated sphere $a_{46}^{3}$ and $b_{46}^{3}$ resonances tabulated in Ref. 2. This limit has already been studied by considering scattering by a sphere having a very thin coating to be a perturbation to uncoated sphere scattering. ${ }^{16}$ In the limit of large $\Delta /$ $A$, the overall particle radii of the composite sphere resonances were found to approach the values for the $n=1.65$ and $n=1.33$ uncoated sphere resonances $a_{46}^{3}$ and $b_{46}^{3}$. This limit has also been studied by perturbation calculations. Between these two limits, we found that if the coated sphere is at the $a_{\ell}^{p}$ or $b_{\ell}^{p}$ resonance and if the core radius is mathematically decreased while the proper coating thickness is added, the $a_{\ell}^{p}$ or $b_{\ell}^{p}$ resonance is able to be maintained.

For the various combinations of coating thicknesses and core radii which produced the $\ell=46$ partial wave resonances, the contribution of the $\ell=46$ partial wave to $\epsilon(r)$ was computed in the core and coating regions and the $\ell=46$ contribution's maximum value $\epsilon^{\max }$ in the interval $0 \leq r \leq B$ was determined. We employ the quantity $\epsilon^{\max }$ as a measure of the size of the internal energy density at resonance. We believe this to be meaningful because, at the $a_{\ell}^{p}$ and $b_{\ell}^{p}$ resonances, the $\ell$ th partial wave contribution to $\epsilon(r)$ dominates the energy density and is proportional to $j_{\ell}^{2}\left(n_{1} k r\right)$ in the core and is proportional to the square of a linear combination of $j_{\ell}\left(n_{2} k r\right)$ and $n_{\ell}\left(n_{2} k r\right)$ in the coating where $n_{\ell}$ are the spherical Neumann functions. At resonance, $\epsilon^{\max }$ is effectively the constant of proportionality of the $\ell$ th partial wave contribution.

For the refractive indices of cases I and II, the maximum interior energy density for the $a_{46}^{3}$ and $b_{46}^{3}$ resonances was calculated and is shown as a function of the coating thickness in Figs. 1 and 2. For a given coating thickness, the $a_{46}^{3}$ and $b_{46}^{3}$ resonances occur at somewhat different core radii. Similarly, the maximum interior energy density was calculated and is shown as a function of coating thickness in Fig. 3 for the $b_{46}^{1}, b_{46}^{2}$ and $b_{46}^{3}$ resonances. These figures show two interesting features. First, when the coating thickness is more than $\sim 45 \%$ of the core radius, the asymptotic thickcoating limit is reached and the composite sphere behaves as if it were an uncoated sphere comprised entirely of coating material. This behavior was also

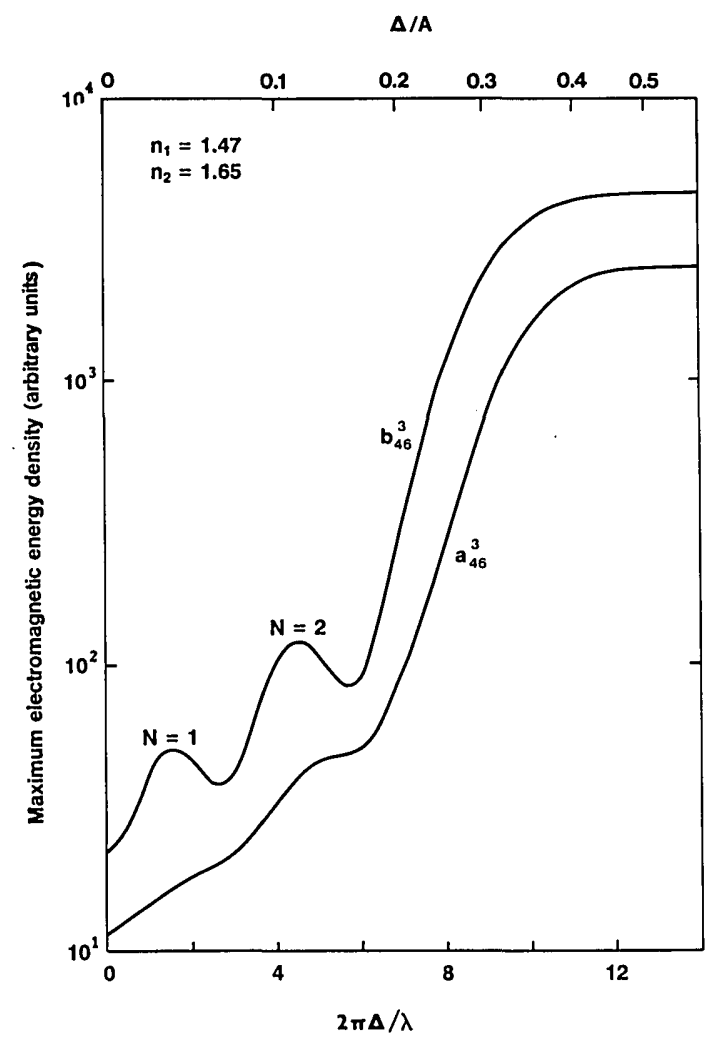

Fig. 1. Maximum value of the contribution of the $\ell=46$ partial wave to the angle-averaged electromagnetic energy density inside a coated sphere for the $a_{46}^{3}$ and $b_{46}^{3}$ scattering resonances as a function of the size parameter associated with the coating thickness $\Delta$. The core and coating refractive indices are $n_{1}=1.47$ and $n_{2}=1.65$ respectively. The $a_{46}^{3}$ and $b_{46}^{3}$ resonances occur at differing core radii. 


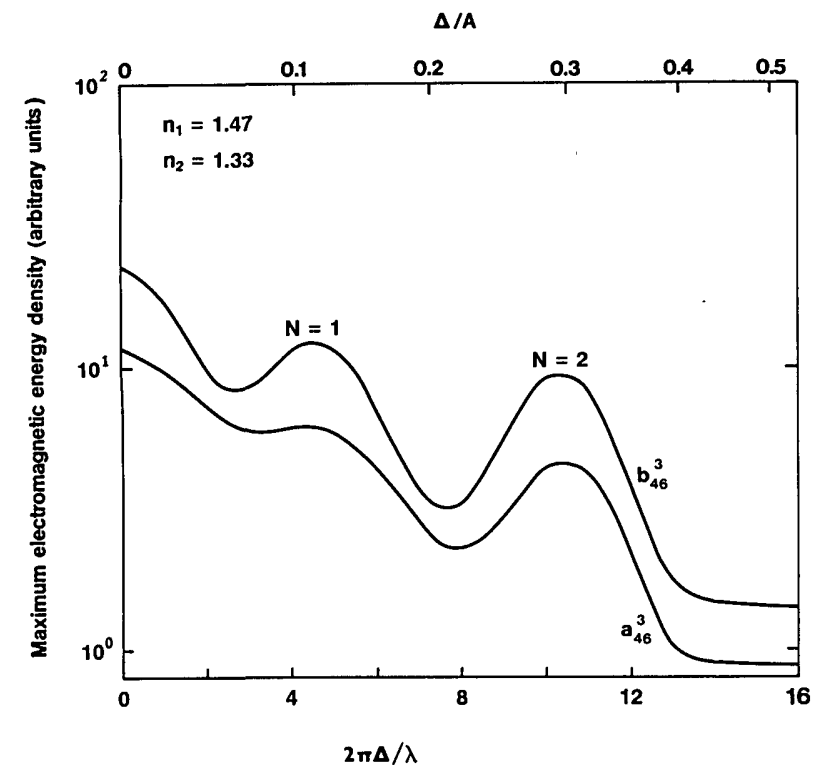

Fig. 2. Maximum value of the contribution of the $\ell=46$ partial wave to the angle-averaged electromagnetic energy density inside a coated sphere for the $a_{46}^{3}$ and $b_{46}^{3}$ scattering resonances as a function of the size parameter associated with the coating thickness $\Delta$. The core and coating refractive indices are $n_{1}=1.47$ and $n_{2}=1.33$ respectively and the units of the energy density are the same as in

Fig. 1. The $a_{46}^{3}$ and $b_{46}^{3}$ resonances occur at differing core radii.

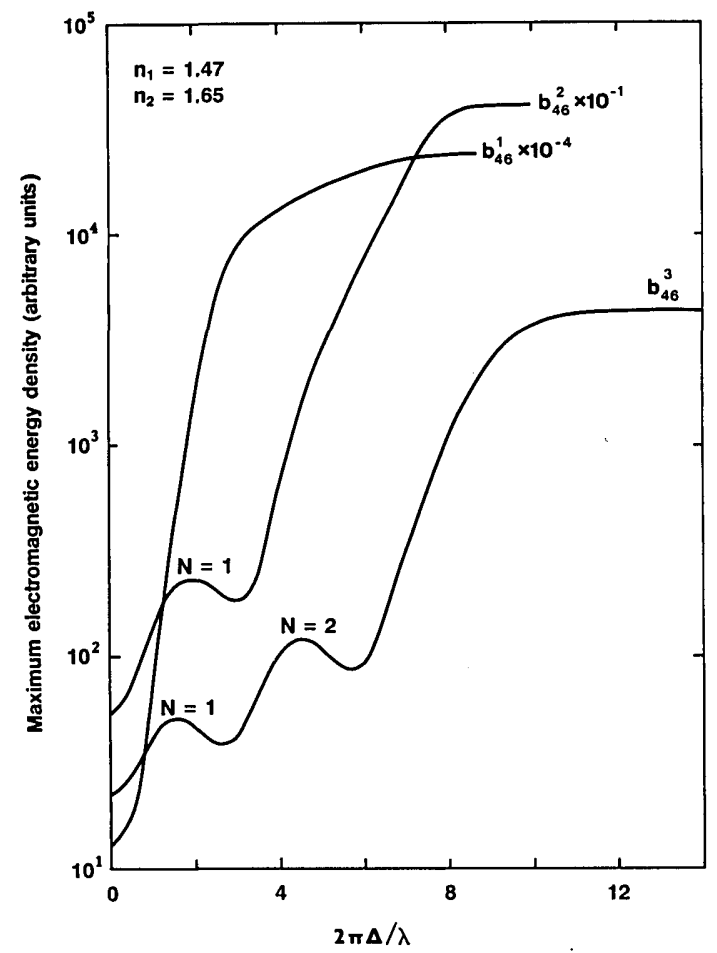

Fig. 3. Maximum value of the contribution of the $\ell=46$ partial wave to the angle-averaged electromagnetic energy density inside a coated sphere for the $b_{46}^{1}, b_{46}^{2}$, and $b_{46}^{3}$ scattering resonances as a function of the size parameter associated with the coating thickness $\Delta$. The core and coating refractive indices are as in Fig. 1 and the units of the energy density are the same as in Figs. 1 and 2. The $b_{46}^{1}$, $b_{46}^{2}$, and $b_{46}^{3}$ resonances occur at differing core radii.

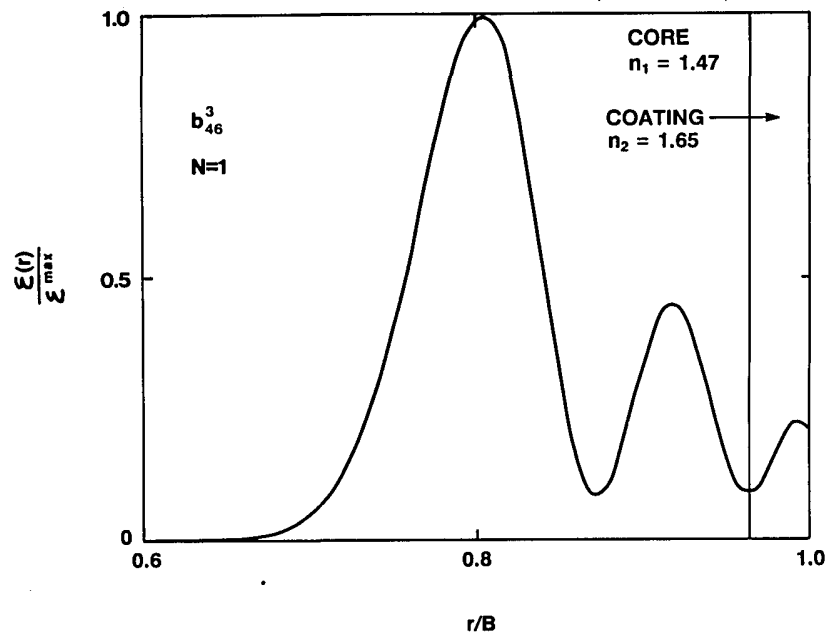

Fig. 4. The contribution of the $\ell=46$ partial wave to the angleaveraged electromagnetic energy density as a function of the distance from the center of the coated sphere for the $N=1$ interference enhancement of the $b_{46}^{3}$ resonance for refractive index case I. The vertical line at $r / B \approx 0.964$ denotes the core-coating interface.

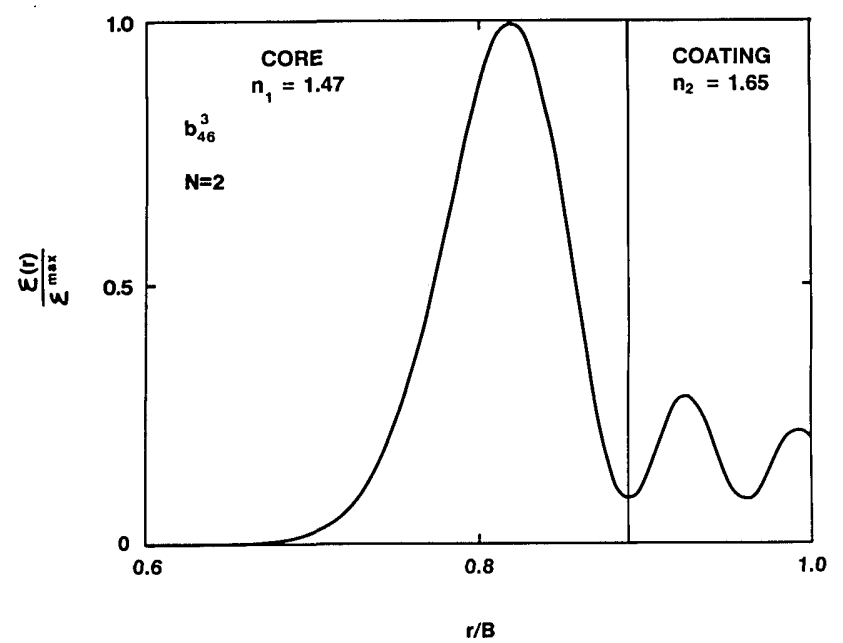

Fig. 5. The contribution of the $\ell=46$ partial wave to the angleaveraged electromagnetic energy density as a function of the distance from the center of the coated sphere for the $N=2$ interference enhancement of the $b_{46}^{3}$ resonance for refractive index case I. The vertical line at $r / B \approx 0.890$ denotes the core-coating interface.

found in Ref. 18 and it results from the near-zero strength of the resonant partial wave's contribution to the interior fields at the core-coating interface for $\Delta \gtrsim$ $0.45 \mathrm{~A}$. The reason is as follows. Strong scattering resonances occur in the partial waves $\ell$ for which $\ell>$ $k B$. If the core-coating interface occurs at

$$
r \lesssim \frac{B}{n_{2}}
$$

the electric and magnetic fields at the interface are small since $j_{\ell}\left(n_{2} k r\right)$ quickly approaches zero as $l>$ $n_{2} k r .35$ For this situation, the resonant wave's contribution to the fields is virtually zero inside the core independent of the core's optical properties. 


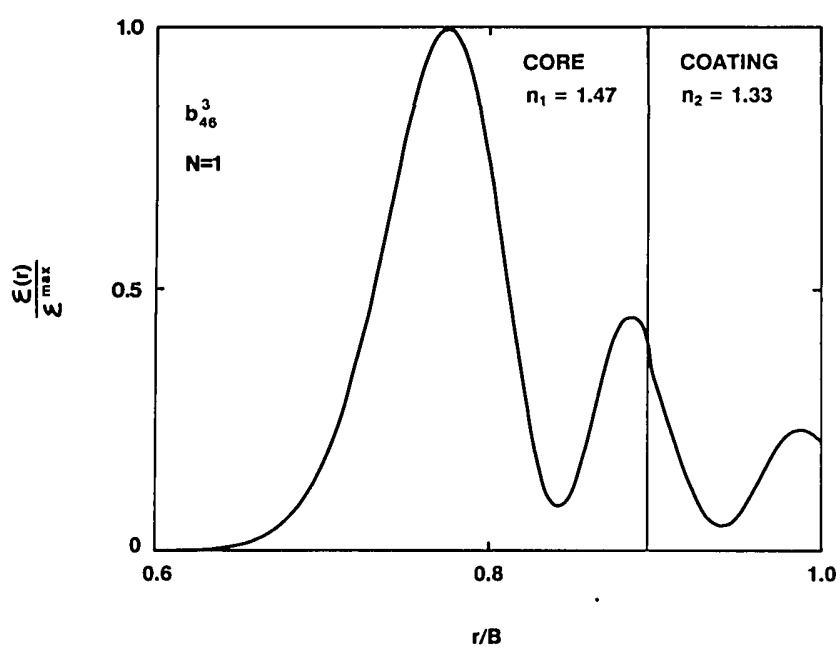

Fig. 6. The contribution of the $\ell=46$ partial wave to the angleaveraged electromagnetic energy density as a function of the distance from the center of the coated sphere for the $N=1$ interference enhancement of the $b_{46}^{3}$ resonance for refractive index case II. The vertical line at $r / B \approx 0.896$ denotes the core-coating interface.

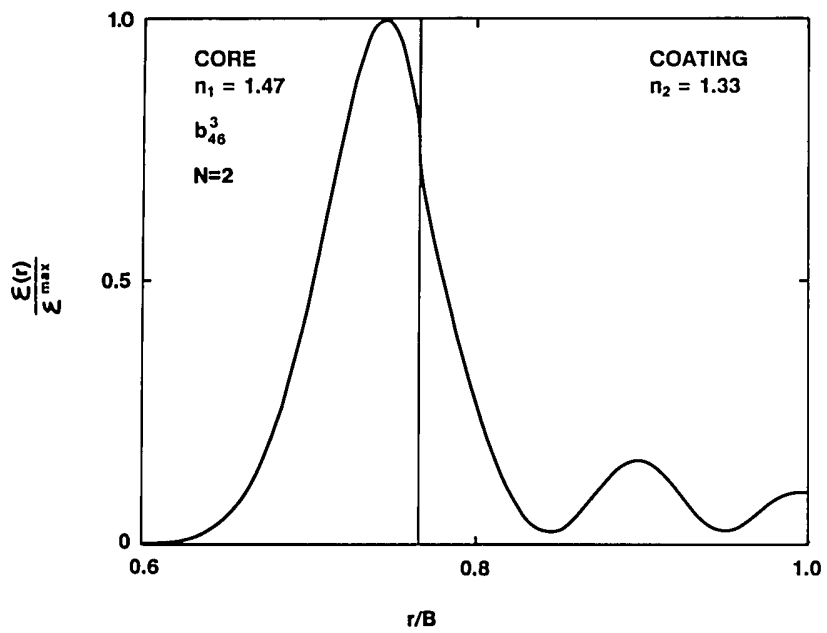

Fig. 7. The contribution of the $\ell=46$ partial wave to the angleaveraged electromagnetic energy density as a function of the distance from the center of the coated sphere for the $N=2$ interference enhancement of the $b_{46}^{3}$ resonance for refractive index case II. The vertical line at $r / B \approx 0.766$ denotes the core-coating interface.

The second interesting feature of Figs. $1-3$ is the presence of $p-1$ enhancements in the interior energy density at the $a_{46}^{p}$ and $b_{46}^{p}$ resonances, labeled by the index $N$ where $1 \leq N \leq p-1$. For the refractive indices of case $I$, the $\ell=46$ partial wave component of the angle-averaged electromagnetic energy density is shown in Figs. 4 and 5 as a function of $r$ for the $N=1$ and $N=2$ enhancements of the $b_{46}^{3}$ resonance respectively. For the indices of case II, the radial dependence of the energy density for the $N=1$ and $N=2$ enhancements of the $b_{46}^{3}$ resonance are shown in Figs. 6 and 7 respectively. These figures indicate that, as was the case for the uncoated sphere, the $a_{\ell}^{p}$ and $b_{\ell}^{p}$ resonances have $(2 p-1) / 4$ radial oscillations of the interi- or fields within the composite sphere. Depending on the coating thickness, any fraction of these oscillations can occur in the core with the remainder occurring in the coating. At the energy density enhancement $N$ for case $I,(p-N) / 2$ oscillations of the internal fields occur in the core and $(2 N-1) / 4$ oscillations occur in the coating. At the energy density enhancement $N$ for case II, $(2 p-1-2 N) / 4$ oscillations occur in the core and $N / 2$ oscillations occur in the coating. These enhancements were not observed in the calculations of Ref. 18 since those authors studied only $p=1$ scattering resonances for which this effect does not occur.

Although the interference enhancements cause an amplification of the interior field strength and energy density, they do not have an effect on the scattering cross section. We have seen that this is mathematically a consequence of the unitarity of the scattering amplitude. At resonance, the partial wave scattering amplitude $a_{\ell}(x)$ or $b_{\ell}(x)$ takes on its upper limit of unity whether or not a quarter-integer of half-integer number of oscillations of the interior fields are present in the core and coating individually. Since all of the exterior scattering quantities depend only on $a_{\ell}(x)$ and $b_{\ell}(x)$, the interference enhancements do not occur in the exterior quantities. They are observable only in experiments which are sensitive to the strength of the interior electric field.

For 1-D systems, a quarter-integer or half-integer number of oscillations of standing waves within an object is a sign that a resonance is being set up within the object. The results of Figs. 4-7 for the scattering resonances of a composite sphere indicate that a similar effect is occurring in the radial direction in the coating and in the core individually.

\section{Reflection from a Composite Thin Film}

There are many analogies between the light scattered from a sphere and the light reflected from a nonabsorbing thin film. ${ }^{36}$ The analogies between these two situations extend to structural scattering resonances and to coated spheres and composite films. A cross section of a thin film is illustrated in Fig. 8. It has thickness $A$ and refractive index $n_{1}$. The light rays $R_{1}$ and $R_{2}$ reflect from the front and rear surfaces of the film. Qualitatively, when these two light rays constructively interfere, the intensity reflectance is maximized. This occurs when

$$
n_{1} k A=(p-1 / 2) \pi
$$

for integer $p \geq 1$ and corresponds to a standing wave with $(2 p-1) / 4$ wavelengths of the fields inside the film.

The intensity reflectance as a function of film thickness may be quantitatively calculated either by matching the boundary conditions of the electric and magnetic fields at the interfaces, see, e.g., Ref. 37, or by summing the infinite series of partial internal reflections from the interfaces, see, e.g., Ref. 38. The boundary condition matching procedure is analogous to the usual derivation of the Mie scattering coefficients, and the internal reflection summation is analo- 


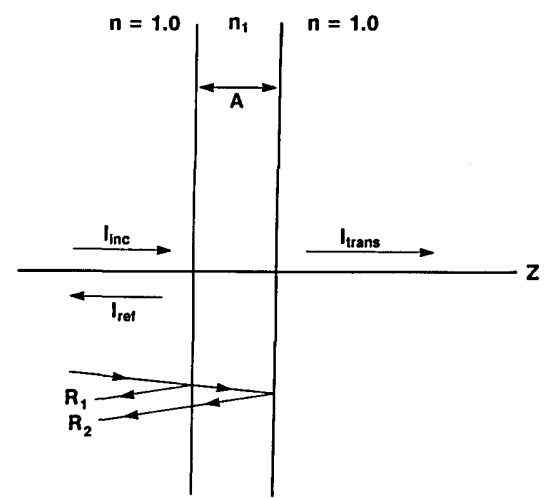

Fig. 8. A thin film and the reflected rays $R_{1}$ and $R_{2}$.

gous to the Debye expansion of the Mie scattering coefficients. Employing either method of calculation, one obtains

$$
\mathscr{R}=\frac{\left(\frac{n_{1}^{2}-1}{2 n_{1}}\right) \sin ^{2} n_{1} k A}{1+\left(\frac{n_{1}^{2}-1}{2 n_{1}}\right)^{2} \sin ^{2} n_{1} k A}
$$

for the intensity reflectance and

$$
|\mathbf{E}(z)|^{2}=\left(\frac{1-\left(\frac{n_{1}^{2}-1}{n_{1}^{2}}\right) \sin ^{2} n_{1} k(z-A)}{1+\left(\frac{n_{1}^{2}-1}{2 n_{1}}\right)^{2} \sin ^{2} n_{1} k A}\right) I_{\text {inc }}
$$

for the square of the magnitude of the interior electric field where $I_{\text {inc }}$ is the incident intensity and $z$ is the distance into the film from the illuminated side. In agreement with the qualitative argument for the $R_{1}$ and $R_{2}$ rays, $\mathscr{R}$ reaches its maximum value when Eq. (12) is satisfied. At this condition, Eq. (14) demonstrates that there are indeed $(2 p-1) / 4$ wavelengths of the fields within the film.

A similar situation occurs for the case of two thin films in contact as illustrated in Fig. 9. Light is incident on the film whose index of refraction is $n_{2}$. Again, the reflectance may be calculated either by matching the boundary conditions at the three interfaces or by summing the infinite series of partial internal reflections from the three interfaces. For the two-film problem, the infinite series of reflections is much more complicated than for the one-film problem. However, the three dominant reflections in the series correspond to the rays $R_{1}, R_{2}$, and $R_{3}$ shown in Fig. 9 which reflect from the front surface of the $n_{2}$ slab, from the $n_{2}-n_{1}$ interface, and from the rear surface of the $n_{1}$ slab respectively. If the relative index is near unity, one qualitatively expects the $R_{2}$ ray to be weak in comparison with the $R_{1}$ and $R_{3}$ rays, and the reflectance to be determined primarily by the interference character of the $R_{1}$ and $R_{3}$ rays. Thus one expects maxima in the reflectance when $R_{1}$ and $R_{3}$ constructively interfere at

$$
n_{1} k A+n_{2} k \Delta=(p-1 / 2) \pi \text {. }
$$

For this condition, there are again $(2 p-1) / 4$ wave-

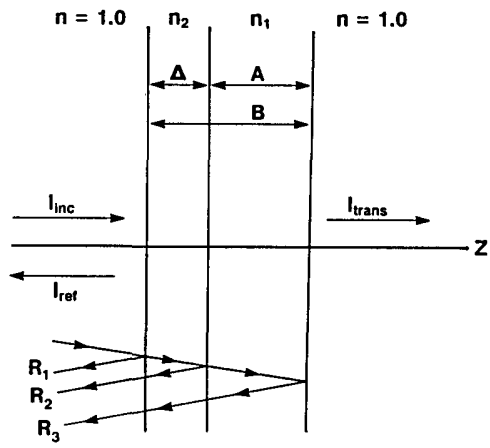

Fig. 9. Two thin films in contact and the reflected rays $R_{1}, R_{2}$, and $R_{3}$.

lengths of the fields within the films. Any fraction of them can be within the $n_{2}$ film with the remainder being in the $n_{1}$ film, depending on the location of the $n_{2}-n_{1}$ interface. Qualitatively one expects the reflectance to attain its largest value when in addition to the $R_{1}$ and $R_{3}$ rays constructively interfering, the $R_{1}$ and $R_{2}$ rays and the $R_{2}$ and $R_{3}$ rays constructively interfere as well. For $n_{2}>n_{1}$, (case I), this extra constructive interference occurs when

$$
\begin{aligned}
& n_{2} k \Delta=(N-1 / 2) \pi \\
& n_{1} k A=(p-N) \pi
\end{aligned}
$$

where $N$ is an integer satisfying $1 \leq N \leq p-1$. For $n_{2}$ $<n_{1}$ (case II), it occurs when

$$
\begin{gathered}
n_{2} k \Delta=N \pi \\
n_{1} k A=(p-N-1 / 2) \pi .
\end{gathered}
$$

The conditions necessary for the production of the interference enhancements of the internal energy density at scattering resonances for the coated sphere problem as described in Figs. 1-7 agree with this qualitative result.

When the two-thin film problem is solved exactly via boundary condition matching method, the reflectance is given by

$$
\begin{aligned}
& \mathscr{R}= 1-8 n_{1}^{2} n_{2}^{2}\left[2 n_{1} n_{2} \cos n_{1} k A \cos n_{2} k \Delta\right. \\
&\left.-\left(n_{1}^{2}+n_{2}^{2}\right) \sin n_{1} k A \sin n_{2} k \Delta\right]^{2}+\left[n_{2}\left(1+n_{1}^{2}\right) \sin n_{1} k A \cos n_{2} k \Delta\right. \\
&\left.\left.+n_{1}\left(1+n_{2}^{2}\right) \cos n_{1} k A \sin n_{2} k \Delta\right]^{2}\right\}^{-1} .
\end{aligned}
$$

For fixed $A$ and variable $\Delta$ the reflectance has a relative maximum for

$$
\tan n_{1} k A \tan n_{2} k \Delta=\frac{2 n_{1} n_{2}}{n_{1}^{2}+n_{2}^{2}} .
$$

For $n_{1}=1.47$ and either $n_{2}=1.65$ or $n_{2}=1.33$, Eq. (21) becomes approximately

$$
\tan \left(n_{1} k A\right) \tan \left(n_{2} k \Delta\right) \approx 1,
$$

which is identical to the qualitative result of Eq. (15). Substituting Eq. (21) and the numerical values of $n_{1}$ and $n_{2}$ into Eq. (20), and allowing both $A$ and $\Delta$ to vary, the largest values of the reflectance are attained at the film thicknesses of Eqs. (16) and (17) or Eqs. (18) and 
(19) depending on whether the indices are of case I or case II respectively.

These results indicate that thin films exhibit a behavior analogous to that found in Sec. III for the interference enhancements of the internal fields at the scattering resonances of a coated sphere, and the thin film results provide a physical interpretation of them. According to the Debye expansion of the scattering amplitudes, a scattering resonance of a homogeneous sphere occurs when all the different multiply internally reflected radial Debye series components for a single partial wave constructively interfere. For a coated sphere, the radial waves reflected from an optically soft core-coating interface are much weaker than those reflected from the coating-air interface. As a result, the constructive interference of the different Debye series components of a single partial wave reflected from the coating-air interface is sufficient to create a scattering resonance of the composite particle. When the core and coating thicknesses are such that quarterinteger or half-integer oscillation standing waves are set up in both the core and coating, the successive internal reflections from both interfaces have the same phase and constructively interfere with each other. This extra constructive interference enhances the energy density within the composite sphere above its value obtained for slightly different resonance conditions.

As was mentioned in the introduction, the resonance spectra of coated spheres and cylinders have a number of potential applications which include diameter and refractive index characterization of optical fibers, the study of surface contamination layers, and the absorption of sunlight by soot particles within cloud droplets. The interference enhancements of coated particle resonances are potentially observable in situations that are sensitive to the strength of the internal fields. The combined results of Figs. 2 and 7 suggest that the $N=p$ - 1 enhancement of the $b_{\ell}^{p}$ resonance for refractive index case II presents an excellent environment in which to investigate the properties of molecules in intense electric fields. For example, Owen, et al. found an order of magnitude increase in the electromagnetic energy density at the surface of a homogeneous fiber at resonance compared to off resonance. ${ }^{31}$ This resulted in an increased fluorescence excitation rate of dye molecules that had been deposited on the surface. As is seen in Figs. 2 and 7, another order of magnitude increase in energy density is potentially available at the core-coating interface at the $N=p-1$ enhancement of the $b_{\ell}^{p}$ resonance. At this condition, molecules deposited at the core-coating interface or diffused into the core material just below the interface experience the largest electromagnetic energy density attainable in resonance spectroscopy.

\section{References}

1. P. Chylek, "Partial-Wave Resonances and the Ripple Structure in the Mie Normalized Extinction Cross Section," J. Opt. Soc. Am. 66, 285-287 (1976).

2. P. Chýlek, J.T. Kiehl, and M. K. W. Ko, "Optical Levitation and Partial-Wave Resonances," Phys. Rev. A18, 2229-2233 (1978).
3. P. Chýlek, J. T. Kiehl, and M. K. W. Ko, "Narrow Resonance Structure in the Mie Scattering Characteristics," Appl. Opt. 17, 3019-3021 (1978).

4. A. Ashkin and J. M. Dziedzic, "Observation of Resonances in the Radiation Pressure on Dielectric Spheres," Phys. Rev. Lett. 38, 1351-1354 (1977).

5. A. Ashkin and J. M. Dziedzic, "Observation of Optical Resonances of Dielectric Spheres by Light Scattering," Appl. Opt. 20, 1803-1814 (1981).

6. H. S. Bennett and G. J. Rosasco, "Resonances in the Efficiency Factors for Absorption: Mie Scattering Theory," Appl. Opt. 17, 491-493 (1978).

7. G. J. Rosasco and H. S. Bennett, "Internal Field Resonance Structure: Implications for Optical Absorption and Scattering by Microscopic Particles," J. Opt. Soc. Am. 68, 1242-1250 (1978).

8. J. F. Owen et al., "Enhancement of Fluorescence Induced by Microstructure Resonances of a Dielectric Fiber," Phys. Rev. Lett. 47, 1075-1078 (1981).

9. R. Thurn and W. Kiefer, "Structural Resonances Observed in Raman Spectra of Optically Levitated Liquid Droplets," Appl. Opt. 24, 1515-1519 (1985).

10. L. M. Folan, S. Arnold, and S. D. Druger, "Enhanced Energy Transfer within a Microparticle," Chem. Phys. Lett. 118, 322327 (1985).

11. H.-M. Tzeng et al., "Laser Emission from Individual Droplets at Wavelengths Corresponding to Morphology-Dependent Resonances," Opt. Lett. 9, 499-501 (1984).

12. J. B. Snow, S.-X. Qian, and R. K. Chang, "Stimulated RamanScattering from Individual Water and Ethanol Droplets at Morphology-Dependent Resonances," Opt. Lett. 10, 37-39 (1985).

13. P. W. Dusel, M. Kerker, and D. D. Cooke, "Distribution of Absorption Centers within Irradiated Spheres," J. Opt. Soc. Am. 69, 55-59 (1979).

14. A. B. Pluchino, "Emissivity Spectra of Composite Microscopic Particles," Appl. Opt. 20, 531-533 (1981).

15. A. B. Pluchino, "Surface Waves and the Radiative Properties of Micron-Sized Particles," Appl. Opt. 20, 2986-2992 (1981).

16. R. Bhandari, "Tiny Core or Thin Layer as a Perturbation in Scattering by a Single-Layered Sphere," J. Opt. Soc. Am. A3, 319-328 (1986).

17. R. Bhandari, "Specific Absorption of a Tiny Absorbing Particle Embedded within a Nonabsorbing Particle," Appl. Opt. 25, 3331-3333 (1986).

18. R. L. Hightower and C. B. Richardson, "Resonant Mie Scattering from a Layered Sphere," Appl. Opt. 27, 4850-4855 (1988).

19. P. Chýlek, "Large-Sphere Limits of the Mie-Scattering Functions," J. Opt. Soc. Am. 63, 699-706 (1973).

20. B. A. Hunter, M. A. Box, and B. Maier, "Resonance Structure in Weakly Absorbing Spheres," J. Opt. Soc. Am. A5, 1281-1286 (1988).

21. J.F. Owen et al., "Determination of Optical-Film Diameter from Resonance in the Elastic Scattering Spectrum," Opt. Lett. 6, 272-274 (1981).

22. H. M. Nussenzveig, "High-Frequency Scattering by a Transparent Sphere. I. Direct Reflection and Transmission," J. Math. Phys. 10, 82-124 (1969).

23. J. A. Lock, "Cooperative Effects Among Partial Waves in Mie Scattering," J. Opt. Soc. Am. A5, 2032-2044 (1988).

24. H. Inada and M. A. Plonus, "The Geometric Optics Contribution to the Scattering from a Large Dense Dielectric Sphere," IEEE Trans. Ant. Prop. AP-18, 89-99 (1970).

25. H. Inada and M. A. Plonus, "The Diffracted Field Contribution to the Scattering from a Large Dense Dielectric Sphere," IEEE Trans. Ant. Prop. AP-18, 649-660 (1970).

26. H. M. Nussenzveig, "High-Frequency Scattering from an Impenetrable Sphere," Ann. Phys. (N.Y.) 34, 23-95 (1965). 
27. H. M. Nussenzveig, "Complex Angular Momentum Theory of the Rainbow and the Glory," J. Opt. Soc. Am. 69, 1068-1079, 1193-1194 (1979).

28. J. R. Probert-Jones, "Surface Waves in Backscattering and the Localization Principle,” J. Opt. Soc. Am. 73, 503 (1983).

29. J. R. Probert-Jones, "Resonance Component of Backscattering by Large Dielectric Spheres," J. Opt. Soc. Am. A1, 822-830 (1984).

30. J. D. Murphy, et al., "A Surface Wave Interpretation for the Resonances of a Dielectric Sphere," IEEE Trans. Ant. Prop. AP-28, 924-927 (1980).

31. J. F. Owen, R. K. Chang, and P. W. Barber, "Internal Electric Field Distributions of a Dielectric Cylinder at Resonance Wavelengths," Opt. Lett. 6, 540-542 (1981).

32. P. Chýlek, "Asymptotic Limits of the Mie-Scattering Characteristics,” J. Opt. Soc. Am. 65, 1316-1318 (1975).
33. P. Chýlek, J. D. Pendleton, and R. G. Pinnick, "Internal and Near-Surface Scattered Field of a Spherical Particle at Resonant Conditions," Appl. Opt. 24, 3940-3942 (1985).

34. C. F. Bohren and D. R. Huffman, Absorption and Scattering of Light by Small Particles (Wiley, New York, 1983) Sec. 8.1, appendix B.

35. A. Messiah, Quantum Mechanics Vol. 1 (Wiley, New York, 1968) p. 490.

36. C. F. Bohren, "Scattering by a Sphere and Reflection by a Slab: Some Notable Similarities," Appl. Opt. 27, 205-206 (1988).

37. R. M. Eisberg, Fundamentals of Modern Physics (Wiley, New York, 1967) section 8.3.

38. J. R. Reitz, F. J. Milford, and R. W. Christy, Foundations of Electromagnetic Theory (Addison-Wesley, Reading, MA, 1979) Sec. 18-5. 